\title{
Metodología para priorizar iniciativas de tecnologías de la información sostenibles
}

\author{
Methodology to prioritize initiatives of sustainable information technologies
}

\author{
Jose Manuel Lamis Rivero ${ }^{1}$, Juan Antonio Plasencia Soler ${ }^{2 *}$, \\ Fernando Marrero Delgado ${ }^{3}$, Miriam Nicado García ${ }^{2}$ \\ ${ }^{1}$ Ministerio de Educación Superior, Cuba \\ ${ }^{2}$ Universidad de las Ciencias Informáticas, Cuba \\ ${ }^{3}$ Universidad Central "Marta Abreu" de Las Villas, Cuba
}

Recibido el 2 de junio de 2018; aceptado el 7 de diciembre de 2018

Disponible en Internet el 11 de diciembre de 2018

\section{Resumen}

En los albores de la economía digital, la sociedad se esfuerza por cumplir los objetivos propuestos para el desarrollo sostenible y pueden ser las tecnologías de la información y las comunicaciones una de las claves para el éxito. Sin embargo, en la actualidad, no se aprovecha correctamente todo su potencial en la solución de las problemáticas asociadas a la sostenibilidad organizacional. La presente investigación propone una metodología para la priorización de iniciativas de las tecnologías y sistemas de información sostenibles en entidades tecnológicas utilizando como principal fundamento matemático el método para la Toma de Decisión Interactiva. Los resultados de la aplicación de la metodología permitieron priorizar las principales prácticas relacionadas con el uso de computadoras portátiles, la comunicación interna y externa de iniciativas para el ahorro de energía y la implementación de sistemas de administración de energía.

Código JEL: Q56, M15, C38

Palabras clave: Sistemas y tecnologías de la información; Sostenibilidad; Método TODIM

\footnotetext{
*Autor para correspondencia Correo electrónico: juanps@uci.cu (J.A. Plasencia Soler).

La revisión por pares es responsabilidad de la Universidad Nacional Autónoma de México. 


\begin{abstract}
At the beginning of the digital economy, the society strives to fulfil the objectives for sustainable development and the information and communication technologies can be one of the keys to success. However, at the present time, all its potential is not properly used to solve the problems associated with organizational sustainability. This research proposes a methodology for the prioritization of sustainable technologies and information systems initiatives in technological entities using the method for Interactive Decision Making as the main mathematical foundation. The results of the application of the methodology allowed prioritizing the main practices related to use of laptops, internal and external communication of initiatives for energy saving and implementation of energy management systems.
\end{abstract}

JEL code: Q56, M15, C38

Keywords: Information systems and technologies; Sustainability; TODIM method

\title{
Introducción
}

En las últimas décadas, las Tecnologías de la Información y las Comunicaciones (TIC) han contribuido significativamente a la innovación, al crecimiento económico y al desarrollo de países y regiones de todo el mundo, pero aún existen reservas en relación al papel que juegan las tecnologías de la información en la construcción de sociedades cada vez más sostenibles.

Las TIC contribuyen a la generación de gases de efecto invernadero y a la contaminación ambiental durante su fabricación, uso y residuos, pero al mismo tiempo son consideradas como una herramienta fundamental para el monitoreo, mitigación y adaptación al cambio climático, según la Comisión Económica para América Latina y el Caribe (CEPAL, 2012). Además, constituyen un elemento facilitador de oportunidades educativas, ya sea a través de la educación masiva, la información en línea o su apoyo la formación de personas (Pattinson, 2017)"container-title":"IFAC-PapersOnLine","collection-title":"20th IFAC World Congress","page":"12938-12943","volume":"50","issue":"1","source":"ScienceDirect","abstract":"The centrality of Information and Communications Technology (ICT.

Según Gartner (2007), las emisiones directas de las Tecnologías de la Información y las Comunicaciones durante su ciclo de vida representan el 2,1\% de las emisiones a nivel global, mientras otros estudios más recientes expresan que las TIC pueden minimizar el impacto ambiental del $97.9 \%$ de las emisiones restantes. De esta manera, las TIC abren una nueva fase en la globalización y constituyen un componente fundamental de las estrategias de cumplimiento del séptimo Objetivo de Desarrollo del Milenio: garantizar la sostenibilidad del medio ambiente (CEPAL, 2012).

Por tales razones, desde hace varios años, la comunidad científica internacional aborda los temas relacionados con el impacto de las TIC en el desarrollo sostenible y específicamente en la sostenibilidad organizacional a través de los Sistemas de Información Verdes, en idioma 
inglés "Green Information Systems" (Green IS) y las Tecnologías de la Información Verdes, "Green Information Technology" (Green IT).

Las Green IS se refieren al desarrollo y la implementación de sistemas de información que contribuyan a la sostenibilidad de los procesos de las empresas; por otra parte las Green IT se enfocan en la eficiencia energética de los medios tecnológicos utilizados para la producción de los servicios informáticos y su reciclaje de manera que no afecte al ambiente (Plasencia-Soler, Marrero-Delgado, Nicado-García y Aguilera-Sánchez, 2017).

Las prácticas de Green IT/IS representan una tendencia reciente, con un amplio campo de aplicación y tienen como meta la eficiencia y eficacia en el diseño, fabricación, desarrollo y uso de las TIC de manera que contribuyan a la sostenibilidad de las organizaciones. Por tales razones se hace necesario identificar y priorizar las iniciativas de Green IT/IS en las organizaciones y especialmente en entidades usuarias de tecnologías de la información (TI).

Por otra parte, para la priorización de alternativas de decisión comúnmente son utilizados métodos multicriterio para el análisis de las decisiones, en inglés "Multiple-Criteria Decision Analysis" (MCDA). Uno de estos métodos, reconocido por tener en cuenta el comportamiento de los decisores, es el método para la Toma de Decisión Interactiva y Multicriterio (TODIM). El TODIM es referenciado por la comunidad científica internacional, como el único método multicriterio fundamentado en la Teoría de los Prospectos, teoría desarrollada por los psicólogos irlandeses Kahneman y Tversky en el año 1979, permitiendo simular el comportamiento de los decisores bajo condiciones de riesgo.

El objetivo de la presente investigación es desarrollar una metodología para la priorización de prácticas Green IT/IS en entidades usuarias de TI que contribuyan a la sostenibilidad organizacional, a través del método para la Toma de Decisión Interactiva y Multicriterio.

Se han estructurado la investigación de la forma siguiente: una primera sección donde se aborda el impacto de las TIC en el desarrollo sostenible y las prácticas o iniciativas de Green IT/IS propuestas por diferentes académicos, así como los fundamentos del método para la Toma de Decisión Interactiva y Multicriterio. En una segunda sección se describe la metodología para la priorización de prácticas de Green IT/IS en entidades de las tecnologías de la información. En un tercer apartado, son enunciados los principales resultados de la aplicación del procedimiento en una entidad usuaria de TI. Finalmente se exponen las conclusiones de la investigación.

\section{La sostenibilidad y las Tecnologías de la Información y las Comunicaciones}

La creciente difusión de las Tecnologías de la Información y las Comunicaciones (TIC) hacia todos los sectores de la sociedad ha llamado la atención de la comunidad científica internacional sobre los efectos negativos y positivos de las TIC en el desarrollo sostenible. 
Por una parte, la producción global de bienes y servicios tecnológicos representa actualmente alrededor del 6,5\% del producto interno bruto (PIB) mundial, y el sector de servicios TIC emplea por si solo a unas 100 millones de personas (UNCTAD, 2017); mientras por otro lado las TIC contribuyen a la generación de gases de efecto invernadero y a la contaminación ambiental durante su fabricación, uso y residuos (CEPAL, 2012).

A decir de Molla (2013) los efectos negativos se asocian principalmente a las emisiones y desechos relacionados con las tecnologías de la información (TI), por lo que lograr que el diseño, la producción, el uso y el reciclaje del producto o servicio tecnológico (Cai, Chen y Bose, 2013) the role of information technology (IT se realice de manera eficiente y efectiva para que no afecte el medioambiente (Murugesan, 2008)"container-title":'IT Professional","page":"24-33","volume":"10","issue":"1","source":"IEEE Xplore","abstract":"In addition to moving itself in a greener direction and leveraging other environmental initiatives, IT could help create green awareness among IT professionals, businesses, and the general public by assisting in building communities, engaging groups in participatory decisions, and supporting education and green advocacy campaigns. Along these lines, tools such as environmental Web portals, blogs, wikis, and interactive simulations of the environmental impact of an activity could offer assistance. Green IT is an economic, as well as an environmental, imperative. Greening IT is and will continue to be a necessity, not an option. Green IT represents a dramatic change in priority in the IT industry. So far, the industry has been focusing on IT equipment processing power and associated equipment spending. It's not been concerned with other requirements such as power, cooling, and data center space. However, going forward, the IT industry will need to deal with all of the infrastructure requirements and the environmental impact of IT and its use. The challenges of green IT are immense; however, recent developments indicate that the IT industry has the will and conviction to tackle our environmental issues headon. The IT sector and users must develop a positive attitude toward addressing environmental concerns and adopt forward-looking, green-friendly policies and practices.",'DOI":"10.1109/ MITP.2008.10",'ISSN":"1520-9202","shortTitle":"Harnessing Green IT","author":[\{“family":"Murugesan","given":"S."\}],"issued":\{“date-parts":[[“2008”,1]]\}\}\}],"schema”:"https://github. com/citation-style-language/schema/raw/master/csl-citation.json"\} se conoce como Green IT (Tecnologías de la Información Sostenibles).

Por otra parte, los efectos positivos se refieren al desarrollo y uso de sistemas de información para mejorar la sostenibilidad ecológica (Dedrick, 2010) mediante la automatización, la informatización y la transformación (Molla, 2009) de los productos y los procesos comerciales de una organización (Watson, Boudreau y Chen, 2010; Jenkin, Webster y McShane, 2011). A este enfoque se le denomina Green IS (Sistemas de la Información Sostenibles).

El estudio y la incorporación a las actividades de las organizaciones usuarios de TI de prácticas relacionadas con las tecnologías y sistemas de información sostenibles es un campo de investigación emergente en la actualidad (Dalvi-Esfahani, Abdul-Rahman y Zakaria, 2015). 
Las investigaciones realizadas por Murugesan (2008); Silva et al. (2013), Esfahani et al. (2015); Nanath y Pillai (2017); motivation towards the adoption (strategic or idealistic/altruisticpermite resumir en la Tabla 1, las principales prácticas de Green IT/IS expuestas en la literatura científica.

Tabla 1

Prácticas para la sostenibilidad de las tecnologías y sistemas de información

Prácticas de Green IT

Centralizar el suministro de equipamiento tecnológico.

Compra de papel y cartuchos ecológicos.

Virtualización de los servidores

Monitorear el consumo de energía de los servidores

Virtualización de las redes

Computación en la nube

Extensión del ciclo de vida de los equipos tecnológicos

Uso de computadores portátiles

Instalación de equipos clientes ligeros

Reciclaje del equipamiento y de sus componentes

Activación de las funciones de gestión de energía en las computadoras

Instalación de aplicaciones para la gestión de energía

Compartir las impresoras multifuncionales en la red

Configuración por defecto de impresión de hojas

por ambas caras en las impresoras

\section{Prácticas de Green IS}

Prácticas de trabajo remoto y encuentro virtuales

Implementación de sistemas de gestión ambiental

Certificación en Sistemas de Gestión Ambiental

Utilización de sistemas logísticos avanzados

Desmaterialización

Establecimiento de objetivos ambientales

Seguimiento de indicadores ambientales

relacionados con las $\mathrm{TI}$

Implementación de un sistema de

administración de energía

Comunicación interna y externa de iniciativas

para el ahorro de energía

Sensores inteligentes para el control

y optimización de los flujos de energía

Seguimiento y análisis los residuos y las emisiones

Fabricación inteligente

Utilización de tecnologías avanzadas

de automatización

Automatización de edificios

Fuente: Elaboración propia.

Una revisión de la literatura científica producida por Chan y Johansson (2014) muestra como la mayoría de las publicaciones sobre las tecnologías y sistemas de información sostenibles se enfocan principalmente en el aspecto ambiental. Precisamente hacia este aspecto se han dirigido las principales críticas del enfoque Green IT/IS.

Las investigaciones sobre la sostenibilidad de las TIC se dirigen a las reducciones del consumo de energía o al desarrollo de aplicaciones informáticas que provean un soporte a la 
mitigación de los impactos ambientales, ignorando las potencialidades para añadir valor a los procesos de negocios, así como el carácter holístico de la sostenibilidad, que incorpora también aspectos económicos, sociales, culturales, jurídicos, tecnológicos, institucionales entre otros.

Por otra parte, la introducción de prácticas asociadas a las tecnologías y sistemas de información sostenibles resultan relevantes para el sector de las TIC, debido a su alta dependencia y uso de las tecnologías de la información (Ruth, 2009)Green IT is an \"in〉" topic. But many problems are unsolved. Information and communications technology (ICT.

Por las razones expuestas anteriormente, es de vital importancia para la gestión sostenible de las entidades usuarias de las tecnologías de la información, la identificación y la priorización de iniciativas de Green IT/IS. Se ha seleccionado para este fin el método para la Toma de Decisión Interactiva y Multicriterio (TODIM).

\section{Método para la Toma de Decisión Interactiva y Multicriterio}

El método para la Toma de Decisión Interactiva y Multicriterio (TODIM) fue introducido a comienzo de la década de 1990 por las publicaciones de Gomes y Lima (1991), (1992) y tiene su base en la Teoría de los Prospectos.

Esta teoría tiene como objetivo evaluar el comportamiento de los seres humanos al tomar decisiones bajo condiciones de riesgo. Los investigadores irlandeses Kahneman y Tversky (1979) observaron que las personas, ante situaciones donde existe la posibilidad de ganar, tienden a ser más conservadores, lo que significa, escoger ganar menos en forma segura a asumir un mayor riesgo para ganar más. Por otra parte, ante escenarios de pérdidas, las personas optan por correr el riesgo de tener pérdidas mayores si existe la posibilidad de no tener pérdidas, a aceptar pérdidas menores.

La Teoría de los Prospectos puede ser representada a través de una función de valor como muestra la Figura 1. Por encima del eje horizontal, se presentan valores positivos o franja de ganancias, y por debajo del mencionado eje está representada la franja de las pérdidas o valores negativos (Rangel y Gomes, 2007).

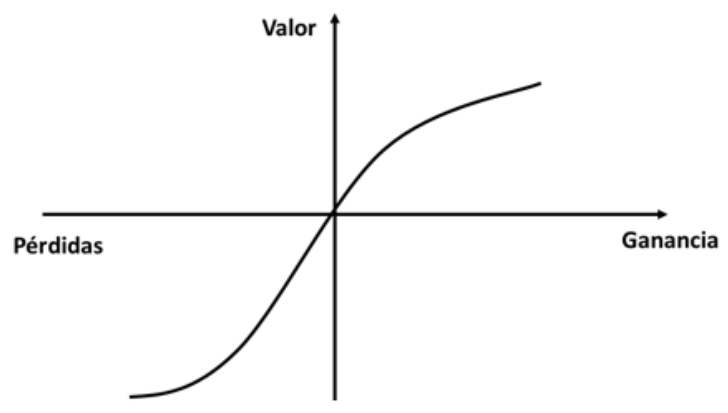

Figura 1. Función de valor Teoría de Prospectos.

Fuente: Elaboración propia con base en Gomes y Lima (1992). 
El procedimiento para su desarrollo puede ser descrito en las etapas siguientes: (i) comparaciones por pares entre los criterios con los juicios de valor expresados; (ii) determinación de un criterio de referencia; (iii) valoración de las alternativas en relación con cada criterio, con el desempeño de cada criterio expresado en una escala ordinal; (iv) formación de una matriz de dominio relativo y; (v) cálculo de las mediciones de los valores globales de cada alternativa y (vi) priorización de todas las alternativas basado en sus valores globales ( Rangel, Gomes y Moreira, 2009; Qin, Liu y Pedrycz, 2017)green supply chain management (GSCM.

Al método TODIM lo distinguen algunas de las características que pueden ser enunciadas en otros MCDA. Es una herramienta técnicamente accesible a los académicos aquellos que incluso no cuenten con conocimientos avanzados sobre los métodos multicriterio. Proporciona una clasificación, a través de la cual, recomienda una decisión determinada. Abarca la utilización de criterios cualitativos y cuantitativos; clasifica los criterios de manera jerárquica y trabaja con la interdependencia de los criterios (Rangel et al., 2009).

No obstante, a decir de Paredes-Frigolett (2016); Yu, Wang y Wang (2016), el TODIM presenta como principales ventajas las siguientes: en primer lugar, tiene cuenta el comportamiento de los decisores en función de la Teoría de los Prospectos. La función de valor está basada en ganancias y pérdidas y no en la posición real de los decisores que es el enfoque convencional utilizado por otros métodos MCDA, basado en la teoría de la utilidad propuesta por von Neumann y Morgenstern (1944). En segundo lugar, el valor potencial de las ganancias y pérdidas, que consigue ajustarse por el factor de las pérdidas, puede utilizarse para reflejar las preferencias de riesgo.

Resultado de estas características y ventajas, es la variada gama de extensiones y modificaciones propuestas por académicos y científicos internacionales en años recientes, así como también la diversidad de campos de aplicación del método, específicamente en temáticas relacionadas con el desarrollo sostenible. Entre estas resaltan, la modelación de la innovación e investigación responsable (Paredes-Frigolett, 2016), la selección de suministradores verdes (Qin et al., 2017; Zhou, Dou, Liao y Tan, 2018)green supply chain management (GSCM, la ubicación de plantas de residuos energéticos con enfoque sostenible (Wu, Wang, Hu, Ke y Li, 2018), la evaluación de ciudades sostenibles (He y Wu, 2017) y la selección de alternativas de energías sostenibles y renovables (Turgut y Tolga, 2018) there has been a remarkable trend toward sustainable and renewable energy sources due to environmental problems and depletion of fossil energy sources. Authorities encourage energy investors to tend this field and consequently many investors develop an energy planning for next decades based on renewable energy sources. At this stage, it is a crucial step to choose energy type and develop a strategic plan based on it. In this study, we aimed to find out the best performing sustainable and/or renewable energy alternative and thus guide decision makers on energy investments. We evaluated four energy power plant types, 
which are solar, wind, hydroelectric and landfilled gas (LFG, por solo mencionar algunos de los estudios más actuales.

Por tales motivos, se ha seleccionado el método TODIM, como parte de la metodología para la priorización de iniciativas de Green IT/IS en la búsqueda de la sostenibilidad organizacional.

\section{Metodología propuesta para la priorización de prácticas de Green IT/IS}

En la investigación se propone una metodología para la priorización de las prácticas de Green IT/IS de una organización a partir de la integración del método TODIM, como se muestra en la Figura 2.

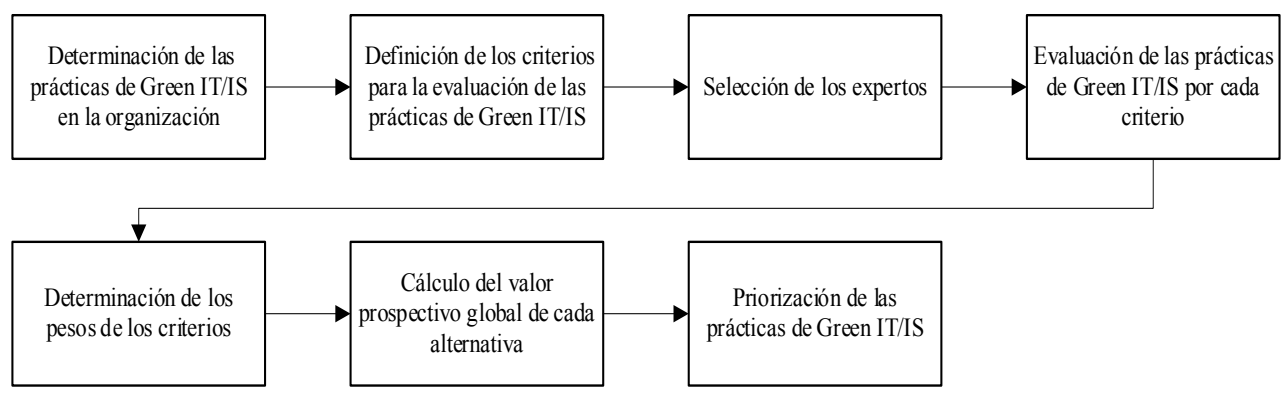

Figura 2. Metodología para la priorización de prácticas de Green IT/IS.

Fuente: Elaboración propia.

A continuación, se describen cada uno de los pasos que componen el procedimiento para la priorización de prácticas de Green IT/IS.

\section{Paso 1. Definición de prácticas de Green IT/IS en la organización}

En este paso se definen las prácticas para la sostenibilidad de las tecnologías y sistemas de información que serán priorizadas por la gerencia. Para este objetivo se puede realizar un diagnóstico de la situación actual de la organización objeto de estudio, identificando las principales cuestiones referentes al diseño, uso, desarrollo y reutilización, tanto del equipamiento tecnológico instalado, como de los servicios y productos informáticos utilizados o desarrollados.

Para este objetivo se pueden utilizar diagnósticos estratégicos realizados anteriormente, datos y registros de las tecnologías de la información y las comunicaciones presentes en la organización, técnicas de trabajo grupal, entre otras fuentes y herramientas. Este paso concluye con un listado de las principales prácticas utilizadas o posibles a utilizar por la organización para la sostenibilidad de las TIC. 


\section{Paso 2. Definir criterios para la evaluación de las prácticas Green IT/IS}

Para este paso, se propone evaluar las prácticas Green IT/IS identificadas, a través del impacto que estas tienen sobre la economía (Eco), la sociedad (Soc) y la ecología (Ecol), teniendo en cuenta uno de los modelos para el desarrollo sostenible más influyentes de la actualidad, el modelo "Resultado Final Triple" en inglés Triple Bottom Line (TBL), tal y como se muestra en la ecuación (1).

Prácticas de Green IT $/ I S=f(E c o$, Soc, Ecol $)$

Una vez definidos los criterios para la evaluación de las prácticas de Green IT/IS, el paso siguiente es seleccionar los expertos que emitirán sus juicios durante el desarrollo de la investigación.

\section{Paso 3. Selección de los expertos}

El trabajo con expertos es de vital importancia en la metodología, son estos los que deberán emitir sus opiniones sobre el impacto de cada una de las prácticas Green IT/IS sobre los criterios seleccionados en el paso anterior. Además, pueden ser utilizados para la determinación de los pesos de cada criterio de decisión.

En este paso, primeramente, se debe calcular la cantidad de expertos que intervienen en la investigación. Por lo que, utilizando un método probabilístico y asumiendo una ley de probabilidad binomial, se calcula el número de expertos a través de la ecuación (2) propuesta en Sarache-Castro, Costa-Salas y Martínez-Giraldo (2015).

$$
n e=\frac{p *(1-p) * k}{i^{2}}
$$

Donde:

ne: número de expertos.

$i$ : nivel de precisión deseado.

$p$ : proporción estimada de errores de los expertos.

$k$ : constante asociada al nivel de confianza elegido.

Luego es necesario identificar dentro de los candidatos a expertos preseleccionados, aquellos con un coeficiente de competencia () alto. Se propone utilizar el método expuesto por Cabero-Almenara y Barroso-Osuna (2013); Michalus, Sarache-Castro y Hernández-Pérez 
(2015) para calcular el coeficiente de competencia y finalmente seleccionar los expertos para el estudio, como muestra la ecuación (3).

$$
K=1 / 2(K c+K a)
$$

Donde:

$K c$ : coeficiente de conocimiento o información.

$K a$ : coeficiente de argumentación o fundamentación.

Con los valores obtenidos se clasifican los expertos en tres grandes grupos: para valores de $\mathrm{K}$ mayores que 0.8 y menores o iguales que 1 , entonces hay influencia alta en todas las fuentes; si K es mayor o igual que 0.7 y menor o igual que 0.8 , entonces hay influencia media en todas las fuentes; finalmente si $\mathrm{K}$ es mayor o igual que $0.5 \mathrm{y}$ menor o iguales 0.7 , entonces existe una influencia baja en todas las fuentes (Cabero-Almenara y Barroso-Osuna, 2013).

\section{Paso 4. Evaluar las alternativas Green IT/IS en cada criterio}

En este paso, los expertos, evalúan cada práctica de Green IT/IS a través de su impacto sobre los criterios definidos anteriormente. Se conforma una matriz de decisión compuestas por las evaluaciones emitidas por los expertos a cada iniciativa en cada criterio, donde en la fila se ubican las iniciativas de Green IT/IS (Green IT IIS $=A_{i} \forall i=1,2, \ldots m$ ) y en las columnas cada uno de los criterios evaluados (Criterios $=C_{j} \forall j=1,2,3$ ) ver ecuación (4).

$$
A=a_{i j}=\left[\begin{array}{cccc}
a_{11} & a_{12} & \cdots & a_{1 j} \\
a_{21} & a_{22} & \ldots & a_{2 j} \\
a_{m 1} & a_{m 2} & \ldots & a_{i j}
\end{array}\right]
$$

Se propone una escala de valores ordinales para la evaluación de las iniciativas de Green IT/ IS en cada criterio, como muestra la Tabla 2. 
Tabla 2

Escala de ponderación para la comparación de los criterios

\begin{tabular}{cc}
\hline Escala ordinal & Código \\
\hline Muy bajo impacto & 1 \\
Bajo impacto & 2 \\
Medio impacto & 3 \\
Alto impacto & 4 \\
Muy alto impacto & 5 \\
\hline
\end{tabular}

Fuente: Elaboración propia.

El coeficiente de variación para cada alternativa () de los juicios emitidos por los expertos debe ser menor al valor de 0.20 .

\section{Paso 5. Determinación de los pesos de los criterios}

En este paso, se determinan los pesos: $\omega_{j} \in[0,1]$ y $\sum_{j=1}^{n} \omega_{j}=1$, asociados a cada uno de los criterios de decisión $\left(C_{j}\right)$. Se pueden utilizar métodos subjetivos y objetivos o una combinación de estos. Se sugiere utilizar el Proceso de la Jerarquía Analítica (PJA) o Método de Saaty (2008), debido a que permite evaluar la consistencia de los expertos en sus valoraciones a través del coeficiente de inconsistencia $(C I)$. Además, con el objetivo de facilitar los cálculos, se recomienda la utilización del software Super Decisión en su versión 2.6.0.

\section{Paso 6. Cálculo del valor prospectivo global de cada alternativa $\left(\xi_{i}\right)$}

En este paso, se utilizaran el conjunto de ecuaciones propuestas por Rangel et al. (2009) para el desarrollo del método TODIM. Primeramente, se calcula la medida de dominancia de la alternativa (i) sobre la alternativa (j) con respecto a cada criterio de decisión, a través de la ecuación (5):

$$
\phi_{c}(\mathrm{i}, \mathrm{j})= \begin{cases}\sqrt{\frac{\mathrm{a}_{r c}\left(\mathrm{~W}_{i c}-\mathrm{W}_{j c}\right)}{\sum_{c=1}^{\mathrm{m}} \mathrm{a}_{r c}}} & \text { si }\left(\mathrm{W}_{i c}-\mathrm{W}_{j c}\right)> \\ 0 & \text { si }\left(\mathrm{W}_{i c}-\mathrm{W}_{j c}\right)= \\ -\frac{1}{\theta} \sqrt{\frac{\left(\sum_{c=1}^{\mathrm{m}} \mathrm{a}_{r c}\right)\left(\mathrm{W}_{i c}-\mathrm{W}_{j c}\right)}{\mathrm{a}_{r c}}} & \text { si }\left(\mathrm{W}_{i c}-\mathrm{W}_{j c}\right)<\end{cases}
$$


$\phi_{c}(\mathrm{i}, \mathrm{j})$ : medida de dominancia de la alternativa (i) sobre la alternativa (j) con respecto a un criterio de decisión.

$\mathrm{a}_{r c}$ : tasa de sustitución o relación de intercambio de los criterios $\mathrm{r}$ y c.

$\mathrm{W}_{i c}-\mathrm{W}_{j c}$ : medidas de valor, o ponderaciones, de las alternativas (i) y (j) para el criterio (c). $\theta$ : factor de atenuación de las perdidas.

Luego se calcula la medida de dominancia general de cada alternativa (i) sobre cada alternativa (j) con respecto al criterio (j) a través de ecuación (6):

$$
\delta(\mathrm{i}, \mathrm{j})=\sum_{\mathrm{c}=1}^{\mathrm{m}} \phi_{c}(\mathrm{i}, \mathrm{j}), \quad \forall(\mathrm{i}, \mathrm{j})
$$

Donde:

$\delta(i, j)$ : representa la medida de dominancia de la alternativa (i) sobre la alternativa (j).

Finalmente, se calcula el valor prospectivo global () de cada alternativa teniendo en cuenta la ecuación (7):

$$
\xi_{i}=\frac{\sum_{j=1}^{n} \delta(i, j)-\min \sum_{j=1}^{n} \delta(i, j)}{\max \sum_{j=1}^{n} \delta(i, j)-\min \sum_{j=1}^{n} \delta(i, j)}
$$

El valor prospectivo global de cada alternativa se encuentra en el intervalo $0 \leq \xi_{\mathrm{i}} \leq$

\section{Paso 7. Priorización de alternativas}

Por último, las alternativas o prácticas de Green IT/IS son priorizadas teniendo en cuenta sus valores prospectivos globales $\left(\xi_{\mathrm{i}}\right)$. Cuanto mayor sea el valor de mejor será la alternativa o práctica de Green IT/IS.

A continuación, se muestran los resultados de la aplicación del procedimiento para la priorización de prácticas Green IT/IS en una entidad de las tecnologías de la información en Cuba.

\section{Resultados de la aplicación del procedimiento}

La dirección de una empresa de productos y servicios informáticos en Cuba necesita determinar las prácticas Green IT/IS que más influyen en el desarrollo sostenible de la organización, 
para trazar líneas de acción encaminadas a su implementación.

Primeramente, se identificaron prácticas de Green IT/IS en la organización, teniendo en cuenta las particularidades de las empresas cubanas del sector y algunas de las más usadas a nivel internacional, como muestra la Tabla 3.

Tabla 3

Prácticas de Green IT/IS identificadas en la organización

\begin{tabular}{ccc}
\hline No & Práctica Green IT/IS & Código \\
\hline 1 & Virtualización de los servidores & Virt_serv \\
2 & Monitorear el consumo de energía de los servidores & Mon_Energ \\
3 & Virtualización de las redes & Virt_redes \\
4 & Computación en la nube & Comp_nube \\
5 & Uso de computadores portátiles & Uso_comp_port \\
6 & Instalación de equipos clientes ligeros & Inst_cliente_lig \\
7 & Compartir las impresoras multifuncionales en la red & Comp_impr \\
8 & Prácticas de trabajo remoto y encuentro virtuales & Pract_trab_remoto \\
9 & Implementación de un sistema de administración de energía & Sist_imple_energ \\
10 & Comunicación interna y externa de iniciativas para el ahorro de energía & Comu_inter_ext \\
\hline
\end{tabular}

Fuente: Elaboración propia.

Seguidamente, se calculó el número de expertos necesarios para la investigación (ne) que fue de 9, con un nivel de precisión del 9\%, una proporción estimada de errores (promedio) del $1 \%$ y para un nivel de confianza del $99 \%$. Luego fueron seleccionados de la cantera de candidatos a expertos los nueve, con mayor coeficiente de competencia.

Los expertos evaluaron el impacto de cada práctica Green IT/IS identificada en cada criterio seleccionado, como se observa en la Tabla 4.

Tabla 4

Muestra las evaluaciones de todas las prácticas Green IT/IS para cada criterio

\begin{tabular}{cccc}
\hline Práctica Green IT/IS & Económico & Social & Ambiental \\
\hline Virt_serv & 4 & 2 & 3 \\
Mon_Energ & 2 & 2 & 5
\end{tabular}




\begin{tabular}{clll} 
Virt_redes & 1 & 1 & 2 \\
Comp_nube & 2 & 3 & 3 \\
Uso_comp_port & 5 & 2 & 5 \\
Inst_cliente_lig & 4 & 1 & 4 \\
Comp_impr & 3 & 2 & 3 \\
Pract_trab_remoto & 1 & 5 & 3 \\
Sist_imple_energ & 1 & 5 & 4 \\
Comu_inter_ext & 1 & 5 & 4 \\
\hline
\end{tabular}

Fuente: Elaboración propia.

A continuación, se calculó el peso $\left(\omega_{j}\right)$ de cada criterio, a través del Método de Saaty. Los expertos seleccionados anteriormente, realizaron las comparaciones pareadas entre los criterios, mediante una escala numérica, indicando cuántas veces un criterio es más importante que otro. Los resultados se muestran en la Tabla 5.

Tabla 5

Pesos de los criterios de decisión

\begin{tabular}{ccccc}
\hline & Económico & Social & Ambiental & \\
\hline Económico & 0.00 & 1.50 & 5.20 & 0.50 \\
Social & 0.67 & 0.00 & 0.18 & 0.20 \\
Ambiental & 0.19 & 5.66 & 0.00 & 0.30 \\
\hline
\end{tabular}

Fuente: Elaboración propia.

Se calculó el coeficiente de inconsistencia $(C I)$ de los juicios emitidos por los expertos y fue menor a 0.10 , valor que indica que hubo consistencia entre estos. Seguidamente se normalizaron los valores, obteniendo, como se muestra en la Tabla 6, la matriz normalizada de iniciativas Green IT/IS evaluadas en cada criterio. La Tabla 6 también muestra la tasa de substitución $\left(\mathrm{a}_{r c}\right)$ entre cada criterio y el criterio de referencia escogido en este caso: el económico. 
Tabla 6

Matriz normalizada de iniciativas Green IT/IS

\begin{tabular}{ccccc}
\hline No & Práctica Green IT/IS & Económico & Social & Ambiental \\
\hline 1 & Virt_serv & 0.167 & 0.071 & 0.083 \\
2 & Mon_Energ & 0.083 & 0.071 & 0.139 \\
3 & Virt_redes & 0.042 & 0.036 & 0.056 \\
4 & Comp_nube & 0.083 & 0.107 & 0.083 \\
5 & Uso_comp_port & 0.208 & 0.071 & 0.139 \\
6 & Inst_cliente_lig & 0.167 & 0.036 & 0.111 \\
7 & Comp_impr & 0.125 & 0.071 & 0.083 \\
8 & Pract_trab_remoto & 0.042 & 0.179 & 0.083 \\
9 & Sist_imple_energ & 0.042 & 0.179 & 0.111 \\
10 & Comu_inter_ext & 0.042 & 0.179 & 0.111 \\
& & 1.000 & 0.400 & 0.600 \\
\hline
\end{tabular}

Fuente: Elaboración propia.

En el paso siguiente corresponde obtener los valores de $\Phi_{c}(i, j)$, según la ecuación (5) expuesta en la sección anterior. Con este objetivo, primeramente, se determinan las diferencias de los pesos $\mathrm{W}_{i c}-\mathrm{W}_{j c}$ como muestra la Tabla 7 para el criterio económico, luego se calcula la medida de dominancia de la alternativa (i) sobre la alternativa (j) con respecto a cada criterio de decisión (Ver Tabla 8).

Tabla 7

Valores $\mathrm{W}_{i c}-\mathrm{W}_{i j}$ de para el criterio económico

\begin{tabular}{ccccccccccc}
\hline & 1 & 2 & 3 & 4 & 5 & 6 & 7 & 8 & 9 & 10 \\
1 & 0.000 & -0.083 & -0.125 & -0.083 & 0.042 & 0.000 & -0.042 & -0.125 & -0.125 & -0.125 \\
2 & 0.083 & 0.000 & -0.042 & 0.000 & 0.125 & 0.083 & 0.042 & -0.042 & -0.042 & -0.042 \\
3 & 0.125 & 0.042 & 0.000 & 0.042 & 0.167 & 0.125 & 0.083 & 0.000 & 0.000 & 0.000 \\
4 & 0.083 & 0.000 & -0.042 & 0.000 & 0.125 & 0.083 & 0.042 & -0.042 & -0.042 & -0.042 \\
5 & -0.042 & -0.125 & -0.167 & -0.125 & 0.000 & -0.042 & -0.083 & -0.167 & -0.167 & -0.167 \\
6 & 0.000 & -0.083 & -0.125 & -0.083 & 0.042 & 0.000 & -0.042 & -0.125 & -0.125 & -0.125 \\
7 & 0.042 & -0.042 & -0.083 & -0.042 & 0.083 & 0.042 & 0.000 & -0.083 & -0.083 & -0.083 \\
8 & 0.125 & 0.042 & 0.000 & 0.042 & 0.167 & 0.125 & 0.083 & 0.000 & 0.000 & 0.000 \\
9 & 0.125 & 0.042 & 0.000 & 0.042 & 0.167 & 0.125 & 0.083 & 0.000 & 0.000 & 0.000 \\
10 & 0.125 & 0.042 & 0.000 & 0.042 & 0.167 & 0.125 & 0.083 & 0.000 & 0.000 & 0.000 \\
\hline
\end{tabular}

Fuente: Elaboración propia. 
Los cálculos de $\mathrm{W}_{i c}-\mathrm{W}_{j c}$ y $\Phi_{c}(\mathrm{i}, \mathrm{j})$ se realizan de igual forma para los criterios social y ecológico.

Tabla 8

Valores de $\Phi_{c}(\mathrm{i}, \mathrm{j})$ para el criterio económico

\begin{tabular}{ccccccccccc}
\hline & 1 & 2 & 3 & 4 & 5 & 6 & 7 & 8 & 9 & 10 \\
1 & 0.000 & -0.408 & -0.500 & -0.408 & 0.144 & 0.000 & -0.289 & -0.500 & -0.500 & -0.500 \\
2 & 0.204 & 0.000 & -0.289 & 0.000 & 0.250 & 0.204 & 0.144 & -0.289 & -0.289 & -0.289 \\
3 & 0.250 & 0.144 & 0.000 & 0.144 & 0.289 & 0.250 & 0.204 & 0.000 & 0.000 & 0.000 \\
4 & 0.204 & 0.000 & -0.289 & 0.000 & 0.250 & 0.204 & 0.144 & -0.289 & -0.289 & -0.289 \\
5 & -0.289 & -0.500 & -0.577 & -0.500 & 0.000 & -0.289 & -0.408 & -0.577 & -0.577 & -0.577 \\
6 & 0.000 & -0.408 & -0.500 & -0.408 & 0.144 & 0.000 & -0.289 & -0.500 & -0.500 & -0.500 \\
7 & 0.144 & -0.289 & -0.408 & -0.289 & 0.204 & 0.144 & 0.000 & -0.408 & -0.408 & -0.408 \\
8 & 0.250 & 0.144 & 0.000 & 0.144 & 0.289 & 0.250 & 0.204 & 0.000 & 0.000 & 0.000 \\
9 & 0.250 & 0.144 & 0.000 & 0.144 & 0.289 & 0.250 & 0.204 & 0.000 & 0.000 & 0.000 \\
10 & 0.250 & 0.144 & 0.000 & 0.144 & 0.289 & 0.250 & 0.204 & 0.000 & 0.000 & 0.000 \\
\hline
\end{tabular}

Fuente: Elaboración propia.

Luego se calcula la medida de dominancia general $\delta(i, j)$ de cada alternativa (i) sobre cada alternativa (j), según la ecuación (6) y sus resultados aparecen en la Tabla 9. Finalmente se calcula el valor prospectivo global $\left(\xi_{i}\right)$ de cada alternativa teniendo en cuenta la ecuación (7), tal y como se muestra en la Tabla 10. Los valores de $\xi_{i}$ permiten al decisor ordenar o priorizar cada una de las alternativas de Green IT/IS identificadas, en función de los criterios: económico, social y ecológico.

Tabla 9

Medida de dominancia general $\delta(i, j)$

\begin{tabular}{ccccccccccc}
\hline & 1 & 2 & 3 & 4 & 5 & 6 & 7 & 8 & 9 & 10 \\
1 & 0.000 & -0.279 & -1.227 & -0.324 & 0.273 & -0.331 & -0.289 & -0.354 & -0.262 & -0.262 \\
2 & -0.226 & 0.000 & -1.238 & -0.346 & 0.250 & -0.523 & -0.286 & -0.573 & -0.447 & -0.378 \\
3 & 0.426 & 0.387 & 0.000 & 0.355 & 0.531 & 0.379 & 0.380 & 0.260 & 0.298 & 0.298 \\
4 & -0.218 & -0.293 & -1.191 & 0.000 & -0.043 & -0.302 & -0.278 & -0.169 & -0.078 & -0.078 \\
5 & -0.719 & -0.500 & -1.527 & -0.846 & 0.000 & -1.016 & -0.839 & -0.861 & -0.735 & -0.735 \\
6 & -0.220 & -0.232 & -0.930 & -0.593 & 0.320 & 0.000 & -0.508 & -0.635 & -0.331 & -0.331
\end{tabular}




\begin{tabular}{ccccccccccc}
7 & 0.144 & -0.160 & -1.135 & -0.204 & 0.333 & -0.187 & 0.000 & -0.262 & -0.171 & -0.171 \\
8 & -0.482 & -0.458 & -1.149 & -0.453 & -0.314 & -0.504 & -0.528 & 0.000 & 0.091 & 0.091 \\
9 & -0.786 & -0.496 & -1.275 & -0.758 & -0.352 & -0.595 & -0.832 & -0.304 & 0.000 & 0.000 \\
10 & -0.786 & -0.496 & -1.275 & -0.758 & -0.352 & -0.595 & -0.832 & -0.304 & 0.000 & 0.000 \\
\hline \multirow{2}{*}{ Total } & -2.868 & -2.529 & -10.949 & -3.926 & 0.647 & -3.674 & -4.012 & -3.202 & -1.634 & -1.566 \\
\hline
\end{tabular}

Fuente: Elaboración propia.

Teniendo en cuenta los resultados de la metodología, la dirección de la organización deberá priorizar las prácticas relacionadas con el uso de computadoras portátiles, la comunicación interna y externa de iniciativas para el ahorro de energía, la implementación de sistemas de administración de energía, la virtualización de los servidores y el monitoreo del consumo de energía de los servidores.

Tabla 10

Valor prospectivo global de cada alternativa

\begin{tabular}{ccc}
\hline $\begin{array}{c}\text { Práctica Green } \\
\text { IT/IS }\end{array}$ & $\xi_{i}$ & Orden \\
\hline Virt_serv & 0.69692383 & 5 \\
Mon_Energ & 0.72615053 & 4 \\
Virt_redes & 0 & 10 \\
Comp_nube & 0.60566604 & 8 \\
Uso_comp_port & 1 & 1 \\
Inst_cliente_lig & 0.62739896 & 7 \\
Comp_impr & 0.59823264 & 9 \\
Pract_trab_re- & 0.66808298 & 6 \\
moto & & 3 \\
Sist_imple_en- & 0.8033047 & 2 \\
erg & & \\
Comu_inter_ext & 0.80921995 & \\
\hline
\end{tabular}

Fuente: Elaboración propia.

Por cada una de las prácticas de Green IT/IS seleccionadas, el centro decisor deberá trazar líneas de acciones, que permitan la implementación de las prácticas priorizadas de Green IT/ IS para la contribución al desarrollo sostenible de la organización. 


\section{Conclusiones}

Las tecnologías de la información y las comunicaciones están llamadas a jugar un papel importante en el complimiento de la Agenda 2030 para el desarrollo sostenible, específicamente para las organizaciones las prácticas de Green IT/IS representan un reto y una oportunidad en la búsqueda de la sostenibilidad no solo en el campo tecnológico sino también en las dimensiones sociales, ecológicas y culturales.

La metodología propuesta tiene su fundamento en la combinación del método para la Toma de Decisión Interactiva y Multicriterio con el trabajo de expertos y el Proceso de la Jerarquía Analítica, lo que provee a la gerencia de un procedimiento robusto y coherente para la determinación de las principales iniciativas de las tecnologías y sistemas de la información sostenibles en una organización.

Los resultados de la aplicación de la metodología propuesta en una entidad de las tecnologías de la información y las comunicaciones permiten a la gerencia concentrarse en las prácticas de Green IT/IS que más afectan la sostenibilidad. Para el caso de estudio seleccionado fueron priorizadas el uso de computadoras portátiles, la comunicación interna y externa de iniciativas para el ahorro de energía, la implementación de sistemas de administración de energía, la virtualización de los servidores y el monitoreo del consumo de energía de los servidores.

Finalmente, la metodología propuesta pudiera ser utilizada para la determinación de otras prácticas o iniciativas para el desarrollo sostenible de las organizaciones, por lo que los trabajos futuros deberán estar dirigidos a su implementación en otros campos de aplicación de las ciencias de la sostenibilidad.

\section{Referencias}


Cabero-Almenara, J., \& Barroso-Osuna, J. (2013). La utilización del juicio de experto para la evaluación de TIC: el coeficiente de competencia experta. Revista de Pedagogía, 65(2), 25-38. https://doi.org/10.13042/brp.2013.65202

Cai, S., Chen, X., \& Bose, I. (2013). Exploring the role of IT for environmental sustainability in China: An empirical analysis. International Journal of Production Economics, 146(2), 491-500. https://doi.org/10.1016/j. ijpe.2013.01.030

CEPAL. (2012). Memoria del seminario realizado en la CEPAL. Presented at the Las TIC y el desarrollo sostenible en América Latina y el Caribe: experiencias e iniciativas de política, realizado en la sede de la Comisión Económica para América Latina y el Caribe (CEPAL), Santiago, Chile: Publicación de las Naciones Unidas.

Chan, H. K. B., \& Johansson, M. (2014). Green IS - a Systematic Literature Review. Disponible en: http://bada. hb.se:80/handle/2320/13797 y Consultado: 13/02/2018

Dalvi-Esfahani, M., Abdul-Rahman, A., \& Zakaria, N. H. (2015). The Status Quo and the Prospect of Green It and Green Is: A Systematic Literature Review. Journal of Soft Computing and Decision Support Systems, 2(1), 18-34.

Dedrick, J. (2010). Green IS: Concepts and Issues for Information Systems Research. Communications of the Association for Information Systems, 27(1). Disponible en: http://aisel.aisnet.org/cais/vol27/iss1/11 y Consultado: $10 / 01 / 2018$

Esfahani, M. D., Rahman, A. A., \& Zakaria, N. H. (2015). Green IT/IS Adoption as Corporate Ecological Responsiveness: An Academic Literature Review. Journal of Soft Computing and Decision Support Systems, 2(1), 35-43.

Gartner. (2007). Gartner Estimates ICT Industry Accounts for 2 Percent of Global CO2 Emissions. Press Release. Disponible en: http://www.gartner.com/it/page.jsp?id=503867 y Consultado: 11/03/2018.

Gomes, L., \& Lima, M. (1991). TODIM: basics and application to multicriteria ranking of pro- jects with environmental impacts. Foundations of Computing and Decision Sciences, 16(4), 113-127.

Gomes, L., \& Lima, M. (1992). From modeling individual preferences to multicriteria ranking of discrete alternatives: a look at prospect theory and the additive difference model. 17, 113-127. Foundations of Computing and Decision Sciences, 17(3), 171-184.

He, X., \& Wu, Y. (2017). City Sustainable Development Evaluation Based on Hesitant Multiplicative Fuzzy Information [Research article]. https://doi.org/10.1155/2017/8306508

Jenkin, T. A., Webster, J., \& McShane, L. (2011). An agenda for 'Green' information technology and systems research. Information and Organization, 21(1), 17-40. https://doi.org/10.1016/j.infoandorg.2010.09.003

Kahneman, D., \& Tversky, A. (1979). Prospect Theory: An Analysis of Decision under Risk. Econometrica, 47(2), 263-291. https://doi.org/10.2307/1914185

Michalus, J. C., Castro, S., William, A., \& Hernández-Pérez, G. (2015). Método de expertos para la evaluación ex-ante de una solución organizativa. Visión de Futuro, 19(1), 0-0.

Molla, A. (2009). Organizational Motivations for Green IT: Exploring Green IT Matrix and Motivation Models. PACIS 2009 Proceedings. Disponible en: https://aisel.aisnet.org/pacis2009/13 y Consultado: 10/02/2018

Molla, Alemayehu. (2013). Identifying IT sustainability performance drivers: Instrument development and validation. Information Systems Frontiers, 15(5), 705-723. http://dx.doi.org/10.1007/s10796-013-9415-z

Murugesan, S. (2008). Harnessing Green IT: Principles and Practices. IT Professional, 10(1), 24-33. https://doi. org/10.1109/MITP.2008.10

Nanath, K., \& Pillai, R. R. (2017). The Influence of Green IS Practices on Competitive Advantage: Mediation Role of Green Innovation Performance. Information Systems Management, 34(1), 3-19. https://doi.org/10.1080/1 0580530.2017.1254436

Paredes-Frigolett, H. (2016). Modeling the effect of responsible research and innovation in quadruple helix innovation systems. Technological Forecasting and Social Change, 110, 126-133. http://dx.doi.org/10.1016/j. techfore.2015.11.001

Pattinson, C. (2017). ICT and Green Sustainability Research and Teaching. IFAC-PapersOnLine, 50(1), 12938-12943. https://doi.org/10.1016/j.ifacol.2017.08.1794 
Plasencia-Soler, J.A., Marrero-Delgado, F., Nicado-García, M., \& Aguilera-Sánchez, Y. (2017). Procedimiento para la priorización de Factores Críticos de Éxito. DYNA, 84(202), 26-34. https://doi.org/10.15446/dyna.v84n202.62084

Qin, J., Liu, X., \& Pedrycz, W. (2017). An extended TODIM multi-criteria group decision making method for green supplier selection in interval type-2 fuzzy environment. European Journal of Operational Research, 258(2), 626-638. https://doi.org/10.1016/j.ejor.2016.09.059

Rangel, L. A. D., Gomes, L. F. A. M., \& Moreira, R. A. (2009). Decision theory with multiple criteria: an aplication of ELECTRE IV and TODIM to SEBRAE/RJ. Pesquisa Operacional, 29(3), 577-590. https://doi.org/10.1590/ S0101-74382009000300007

Rangel, L. A., \& Gomes, L. F. A. (2007). Determinação do valor de referência do aluguel de imóveis residenciais empregando o Método TODIM, 27. http://dx.doi.org/10.1590/S0101-74382007000200009

Ruth, S. (2009). Green IT More Than a Three Percent Solution? IEEE Internet Computing, 13(4), 74-78. https:// doi.org/10.1109/MIC.2009.82

Saaty, T. L. (2008). Decision making with the analytic hierarchy process. Int. J. Services Sciences, 1(1), 83-98. https://doi.org/10.1504/ijssci.2008.017590

Sarache-Castro, W. A., Costa-Salas, Y. J., \& Martínez-Giraldo, J. P. (2015). Environmental performance evaluation under a green supply chain approach. DYNA, 82(189), 207-215. http://dx.doi.org/10.15446/dyna.v82n189.48550

Silva, L. C., Poleto, T., Moura, J. A. d, Daher, S. F. D., \& Costa, A. P. C. S. (2013). Group Decision Approach to Adopt Green IT Practices Based on S.W.O.T Analysis. In 2013 IEEE International Conference on Systems, Man, and Cybernetics (pp. 747-751). https://doi.org/10.1109/SMC.2013.132

Turgut, Z. K., \& Tolga, A. Ç. (2018). Fuzzy MCDM Methods in Sustainable and Renewable Energy Alternative Selection: Fuzzy VIKOR and Fuzzy TODIM. In Energy Management-Collective and Computational Intelligence with Theory and Applications (pp. 277-314). Springer, Cham. https://doi.org/10.1007/978-3-319-75690-5_13

UNCTAD. (2017). Informe sobre la Economía de la Información 2017. Digitalización Comercio y Desarrollo. Panorama General. Ginebra, Zuisa: Naciones Unidas.

von Neumann, J., \& Morgenstern, O.,. (1944). Theory of Games and Economic Behavior. Princeton: Princeton University Press.

Watson, R. T., Boudreau, M.-C., \& Chen, A. J. (2010). Information Systems and Environmentally Sustainable Development: Energy Informatics and New Directions for the IS Community. MIS Quarterly, 34(1), 23-38. https://doi.org/10.2307/20721413

Wu, Y., Wang, J., Hu, Y., Ke, Y., \& Li, L. (2018). An extended TODIM-PROMETHEE method for waste-to-energy plant site selection based on sustainability perspective. Energy, 156, 1-16. https://doi.org/10.1016/j.energy.2018.05.087

Yu, S., Wang, J., \& Wang, J. (2016). An extended TODIM approach with intuitionistic linguistic numbers. International Transactions in Operational Research, 25(3), 781-805. https://doi.org/10.1111/itor.12363

Zhou, Z., Dou, Y., Liao, T., \& Tan, Y. (2018). A Preference Model for Supplier Selection Based on Hesitant Fuzzy Sets. Sustainability, 10(3), 659. http://dx.doi.org/10.3390/su10030659 